\title{
Simplifying the Assessment of Stereognostic Ability of the Tongue in Elderly Subjects Using Six Selected Test Pieces
}

\section{Shigenori Kawagishi ${ }^{1 *}$, Toshiko Tanaka1, Megumi Shimodozono ${ }^{2}$ and Kenichi Yoshino}

${ }^{1}$ Department of Health Improvement, Kyushu Dental University, Kitakyushu, Japan

${ }^{2}$ Department of Rehabilitation and Physical Medicine, Graduate School of Medical and Dental Sciences, Kagoshima University, Kagoshima, Japan

${ }^{3}$ Department of Oral Health Management, School of Oral Health Sciences, Kyushu Dental University, Kitakyushu, Japan

\begin{abstract}
This study aimed to develop a simple method to assess the stereognostic ability of the tongue in elderly. The stereognostic ability was assessed by placing 6 differently-shaped test pieces in the oral cavity and assessing the subjects' ability to identify the shape using their tongues. These test pieces were selected from 20 test pieces that were previously used for detailed analysis of stereognostic ability of the tongue. Stereognostic ability of the tongue in elderly subjects was compared with young adults. In total, 188 out of 198 young adults ( $94.9 \%$ of subjects), 26 out of 60 elderly $(43.3 \%)$ not receiving care, and 1 out of 18 elderly $(5.5 \%)$ receiving care could identify all 6 test pieces correctly. The most misidentified piece was a rectangular test piece and it was recognized as the test piece of the same size without corners, and vice versa.
\end{abstract}

When 35 elderly who were not receiving care were assessed using either 6 or 20 test pieces, the number of correct responses for the 6 test pieces showed a significant positive correlation with those for 20 test pieces $(p<0.001)$.

These results indicate that the method using just 6 test pieces is a simple yet effective approach to assess stereognostic ability of the tongue and that it could be used in future assessments with larger and more diverse patient populations.

Keywords: Oral sensation; Tongue; Stereognosis; Test pieces; Aging; Care

\section{Introduction}

Oropharyngeal swallowing disorders are a common problem, especially in the elderly or patients with cerebrovascular disorders and can cause a decline in their quality of life, aspiration pneumonia, dehydration, malnutrition, and even death [1-4]. Assessments and treatments for patients with oropharyngeal dysphagia have thus become important due to a progressive increase in the elderly population [5].

The tongue plays important roles in mastication and swallowing [6]. The functions of the tongue in oropharyngeal swallowing have been evaluated primarily in terms of motor impairment, such as active range of tongue motion, symmetry at rest, during protrusion, lateralization and elevation, and strength in protrusion, or by items such as bolus formation, tongue-to-palate contact, residue in the oral cavity, premature bolus loss, and oral transit time obtained with video fluoroscopic studies [7-9]. Hans et al. [8] studied through video fluoroscopy, prognostic factors that affect the recovery of swallowing function of stroke patients and reported that scores for functions of the tongue were very high, 30-42, in the video fluoroscopic dysphagia scale with a sum of 100. It is well known that sensory feedback is important in motor control and learning [10]. During reaching movements, the application of visual feedback supports the somatic sensory (proprioceptive) feedback. However, somatic sensation is the only feedback source in the oral cavity during the oral phase of oropharyngeal swallowing. The size and shape of an intraoral bolus of food provides oral sensory information important for oropharyngeal swallowing and the tongue is responsible for oral stereognosis [11-16]. However, the relationships between decrease in oral sensory and tongue motor disability or dysphasia and rehabilitation focused on the recovery of sensory functions have not been thoroughly examined. With the aim of evaluating these relationships, we previously showed that a test that involved identifying 20 different shapes of test pieces in the mouth was effective for detailed analysis of stereognostic ability of the tongue [17] However, completing the test using 20 test pieces seemed difficult for many elderly. This difficulty prompted us to simplify the method of evaluation for further research, such as assessments in a larger number of elderly, in patients with cerebrovascular disorders, and in clinical application such as oral rehabilitation.

In this study, we investigated the differences of stereognostic activity of the tongue between young adults and elderly using 6 test pieces selected from the 20 test pieces and showed that only 6 test pieces can sufficiently and effectively assess the difference in the activity in the above two groups and even in healthy elderly and elderly receiving care.

\section{Materials and Methods}

\section{Subjects}

The subjects were composed of three groups. Two groups were198 young adults (young adult group; students of Kyushu Dental University; 115 males, 83 females; mean age: 24.4 years; age range: 23 to 31 years) and 60 elderly (healthy elderly group; residents of two nursing homes who were not receiving nursing care; 14 males, 46 females; mean age: 80.1 years; age range: 65 to 92 years), without masticatory or swallowing

*Corresponding author: Shigenori Kawagishi, Department of Health Improvement, Kyushu Dental University, 2-6-1 Manazuru, Kokurakita-ku, Kitakyushu 803-8580, Japan, Tel: (+81)-93-285-3039; Fax: (+81)-93-582-6000; E-mail: kawagisi@kyu-dent.ac.jp

Received July 02, 2013; Accepted October 17, 2013; Published October 19 2013

Citation: Kawagishi S, Tanaka T, Shimodozono M, Yoshino K (2013) Simplifying the Assessment of Stereognostic Ability of the Tongue in Elderly Subjects Using Six Selected Test Pieces. Aging Sci 1: 111. doi: 10.4172/2329-8847.1000111

Copyright: (c) 2013 Kawagishi S, et al. This is an open-access article distributed under the terms of the Creative Commons Attribution License, which permits unrestricted use, distribution, and reproduction in any medium, provided the original author and source are credited. 

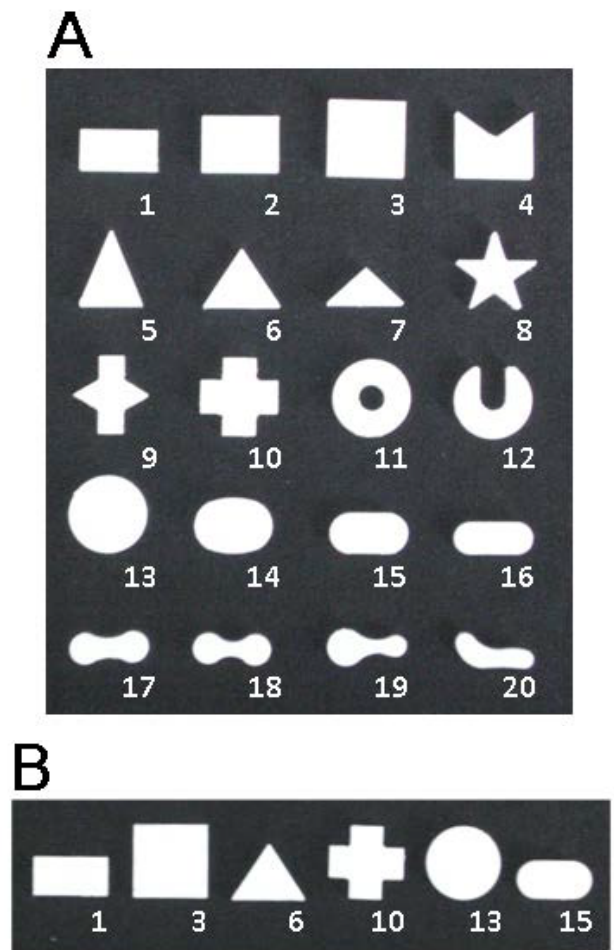

Figure 1: The 20 and 6 different shapes of test pieces used in the 20PT (A) and $6 \mathrm{PT}(\mathrm{B})$ test.

dysfunction. The other group included 18 subjects receiving care at their own home (cared elderly group; 4 males, 14 females; mean age: 71.3 years; age range: 56 to 91 years). This group received care for their daily activities provided by workers who came to their homes under the public nursing-care insurance system. Their scores for necessity of care were 1-3; the highest care score is 5 , which is the score for people confined to bed. None of them showed obvious masticatory or swallowing dysfunction although some needed help in eating.

Approval for the study protocol was obtained from the ethics committee of Kyushu Dental University. Written consent was obtained from all subjects following sufficient explanation of the purpose and content of the research.

\section{Assessment of stereognostic ability of the tongue}

To assess the stereognostic ability of the tongue, we used a method whereby the shape of intraoral test pieces was evaluated, as previously described [17]. We assessed the stereognostic ability using two methods in which several polyethylene test pieces (13-mm length, 2 -mm thickness) were used. The test pieces are shown in Figure 1. The methods using 20 and 6 different shapes of test piece are referred to as the $20 \mathrm{TP}$ and $6 \mathrm{TP}$ tests, respectively. The test pieces were based on shapes developed by the American National Institute of Health [18]. A fine thread (diameter: $0.2 \mathrm{~mm}$ ) was attached to each test piece to prevent choking. The 6 test pieces were selected from the 20 test pieces on the basis of ability to detect differences in simple but different shapes, similar but different-sized shapes, and similar but absence or presence of angles: No. 1 and 3 from the polygonal-shaped group, No. 13 and 15 from the convex-shaped group with no corners and ends swelling convexly, and No. 6 and 10, which are very different from No. $1,3,13$ and 15 .
The test procedure was as follows: The test was carried out between 2:00-5:00 p.m. in a quiet environment where the subjects were seated comfortably in an upright position. With the eyes closed, the subjects put out their tongues, and one of 20 test pieces in the 20TP test (6 test pieces in the 6TP test) was randomly selected and placed on the middle of the tongue. The subjects were asked to move the piece in their mouths without it touching the teeth or gums to determine its shape, and choose the corresponding shape from pictures of the 20 variously shaped test pieces ( 6 test pieces in the 6TP test). The test was completed when all 20 test pieces ( 6 test pieces in the 6TP test) had been evaluated in this manner. It was possible for the subjects to choose the same test piece multiple times in their responses. The subjects were not informed whether or not their responses were correct. In the 20TP test the test was repeated four times for the same individual, and in the 6TP test, one time. The 20TP and 6TP tests in the same individual were carried out immediately without rest period. Analyses of data were performed to determine the stereognostic ability of the tongue, because it had already been shown that covering the palate does not affect oral stereognostic ability [19].

\section{Statistical analysis}

A chi-square test was utilized for examining the association between the number of subjects with all correct answers and groups. A one-way ANOVA test and Tukey's HSD were used for multiple comparisons among three or more independent groups after a Bartlett test. p-values less than 0.05 were considered significant.

\section{Results}

\section{Assessment of stereognostic ability of the tongue in young} adults

In the 6TP test, 188 out of 198 young adults (94.9\% of subjects) could identify all 6 test pieces correctly (Table 1). The numbers of correct responses of the 8 and 2 subjects out of the 10 subjects $(5$ males, 5 females) who could not identify all test pieces perfectly were 5 and 4 , respectively. Figure 2 shows the actual test piece inserted into the mouth

\begin{tabular}{|l|c|c|c|}
\hline \multirow{2}{*}{ Group } & \multicolumn{3}{|c|}{ Number of subjects } \\
\cline { 2 - 4 } & All $\mathbf{6}$ correct answers & 1 or more incorrect answers & Total \\
\hline Young adult & 188 & 10 & 198 \\
\hline Healthy elderly & 26 & 34 & 60 \\
\hline Cared elderly & 1 & 17 & 18 \\
\hline
\end{tabular}

Table 1: Number of subjects with all correct answers and one or more incorrect answers in the three groups.

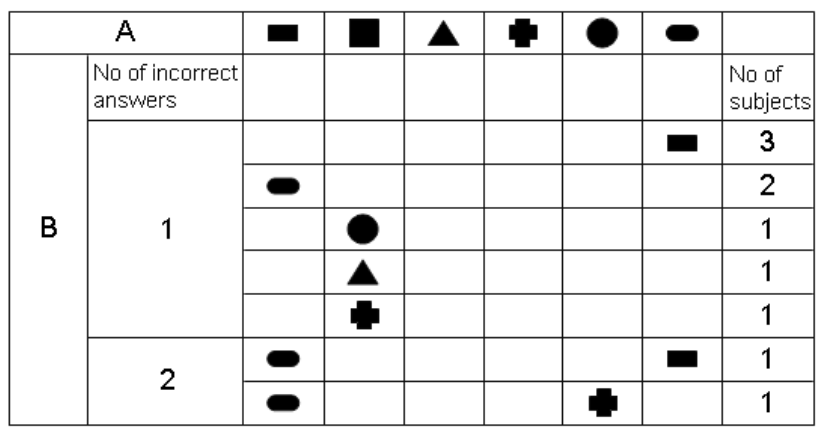

Figure 2: Misidentified shapes by young adults. A (Horizontal): The actual test piece inserted into the mouth; $\mathrm{B}$ (Vertical): The test piece chosen by subjects. 
Citation: Kawagishi S, Tanaka T, Shimodozono M, Yoshino K (2013) Simplifying the Assessment of Stereognostic Ability of the Tongue in Elderly Subjects Using Six Selected Test Pieces. Aging Sci 1: 111. doi: 10.4172/2329-8847.1000111

and the test piece chosen incorrectly by the 10 subjects. In most cases of misidentification, No. 1 was misidentified as the similar No. 15 , and vice versa. The number of correct responses of these 10 subjects in the 20TP test was from 10.5 to 17.5 (mean \pm SD: $13.3 \pm 2.5$ ), and the number of correct responses of 198 young adults in the $20 \mathrm{TP}$ test was $16.6 \pm 2.2$.

Assessment of stereognostic ability of the tongue in healthy elderly

In the $6 \mathrm{TP}$ test, 26 out of 60 healthy elderly (43.3\%) could identify all 6 test pieces (Table 1). The subjects who made 1, 2, 3, 5 and 6 misidentifications in the $6 \mathrm{TP}$ test were $17,12,3,1$ and 1 out of the 34 subjects, respectively. Figure 3 shows the actual test piece inserted into the mouth and the test piece chosen incorrectly by the 34 subjects. Many subjects, 20 elderly, misidentified No.15 as No.1.

\section{Assessment of tongue stereognostic ability in cared elderly}

In the 6TP test, only one out of 18 cared elderly (5.5\%) could identify all 6 test pieces (Table 1). The test pieces chosen incorrectly by the 17 subjects are shown in Figure 4. The most cases of misidentification were between No. 1 and 15, the same as the other two groups. In addition, there were various kinds of misidentifications, such as the case of misidentification of No.13. Six out of 8 healthy elderly misidentified No.13 as No.3 (Figure 3). On the other hand, this misidentification occurred in only 2 out of 7 cared elderly and there were misidentifications as No.6 or No.10, which were not observed in the healthy elderly (Figure 4).

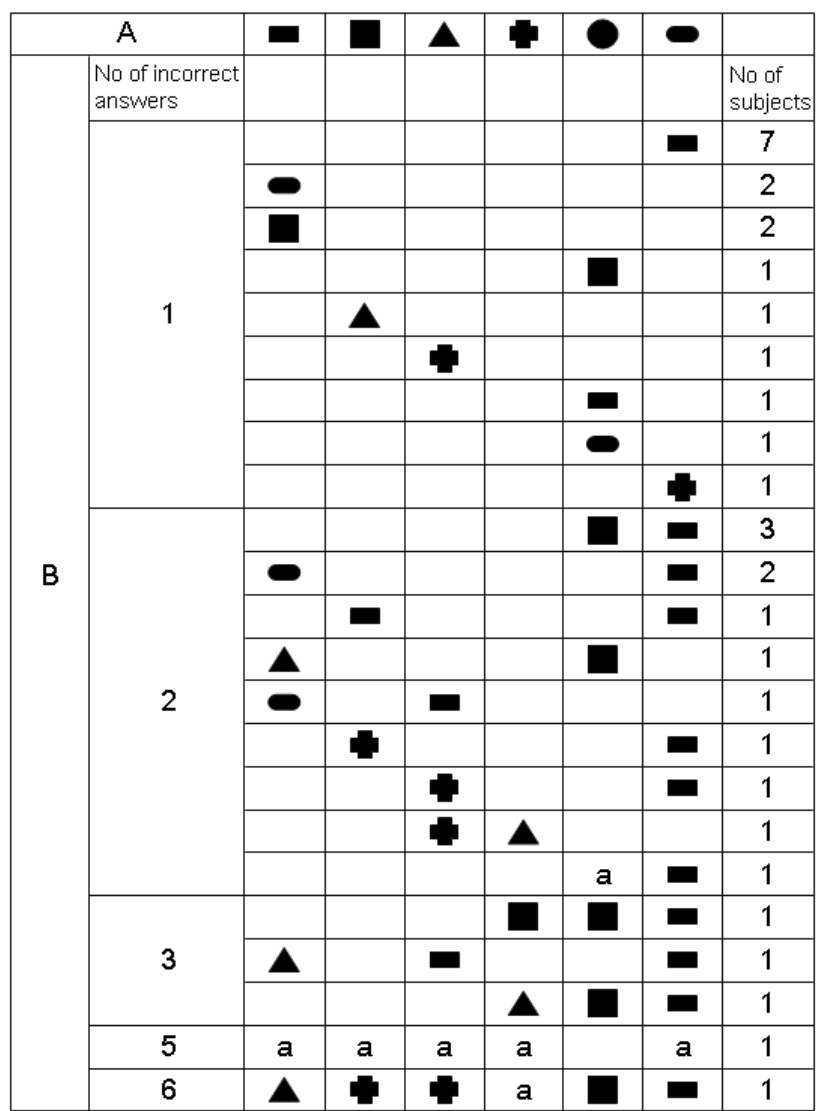

Figure 3: Misidentified shapes by elderly who were not receiving care. A (Horizontal): The actual test piece inserted into the mouth; B (Vertical): The test piece chosen by subjects. a: Subject could not recognize the shape.

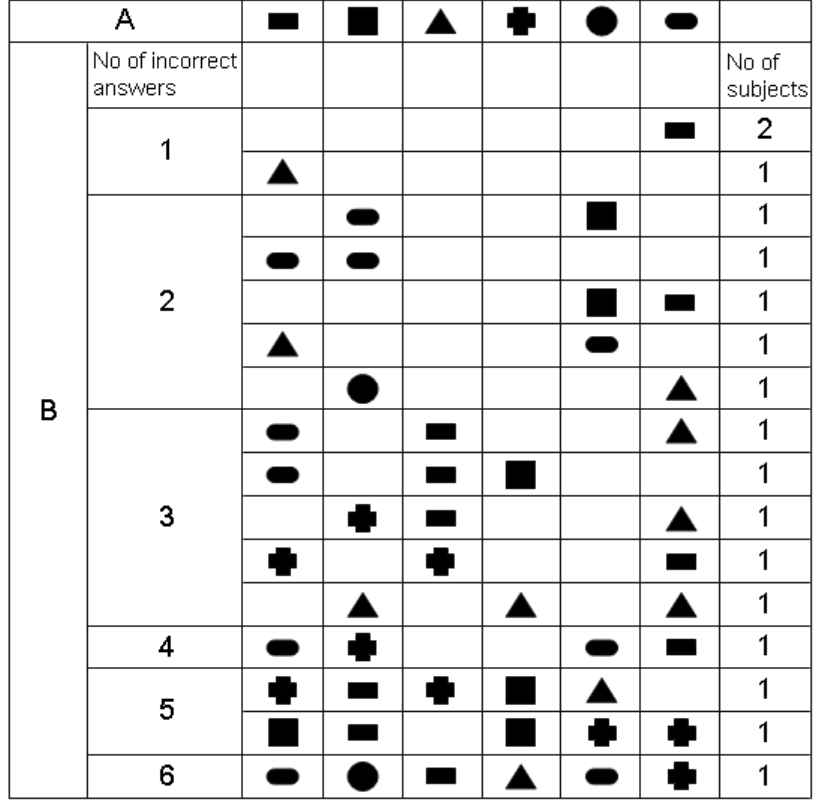

Figure 4: Misidentified shapes by subjects receiving care. A (Horizontal): The actual test piece inserted into the mouth; B (Vertical): The test piece chosen by subjects.

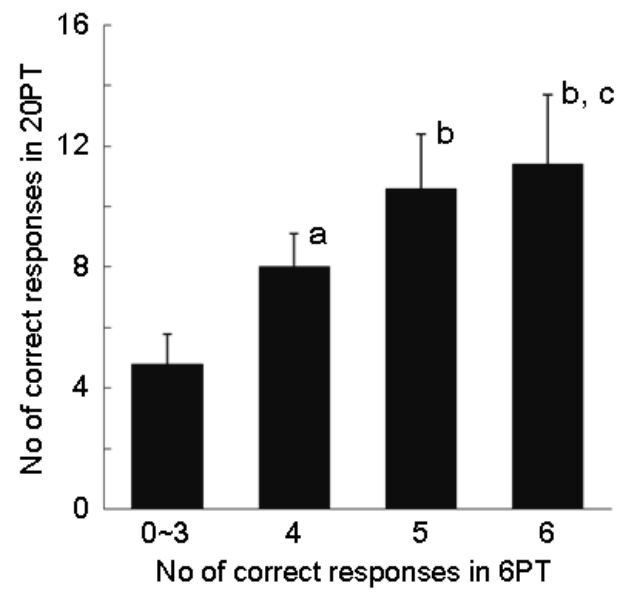

Figure 5: Correlation between number of correct responses in the 20PT and 6PT tests performed by elderly subjects who were not receiving care. $\mathrm{P}<0.05, \mathrm{~b}: \mathrm{P}<0.01$ versus $0 \sim 3$ group; $\mathrm{c}$ : $\mathrm{P}<0.02$ versus 4 group

There was a significant difference in the number of subjects who could correctly identify all 6 test pieces in the young adult, healthy elderly and cared elderly groups $(\mathrm{p}<0.001)$ (Table 1$)$.

Relationship between number of correct responses in 20PT and 6PT tests in healthy elderly

Stereognostic ability of the tongue in 35 (residents of one of two nursing homes, 6 males, 29 females; mean age: 81.8 years) out of 60 healthy elderly was also assessed using the $20 \mathrm{TP}$ test. The number of correct responses in the 20TP test was $9.6 \pm 2.5$ when the subjects were tested 4 times. In the $6 \mathrm{TP}$ test, 16 out of 35 subjects $(45.7 \%)$ could identify all 6 test pieces perfectly. Figure 5 shows the relationship between the number of correct responses in the 20TP and 6TP tests 
of the subjects. In the comparison of the $20 \mathrm{TP}$ and $6 \mathrm{TP}$ tests, the values obtained from the first test in the 20TP test, not the mean of the 4 tests, were used. The numbers of correct responses in the 20TP test of the subjects whose numbers of correct responses were $0 \sim 3,4$, 5 and 6 in the $6 \mathrm{TP}$ test were $4.8 \pm 1.0,8.0 \pm 1.1,10.6 \pm 1.8$, and $11.4 \pm$ 2.3 , respectively. The number of correct responses in the 20PT test was proportional to that in the $6 \mathrm{PT}$ test, showing a significant difference in one-way ANOVA $(\mathrm{p}<0.001)$. The group with a perfect score on $6 \mathrm{TP}$ showed significantly higher scores in the 20TP test than the groups with $6 \mathrm{TP}$ scores of $0-3$ or 4 . The numbers of correct responses in 20TP for those with scores of 4 and 5 in $6 \mathrm{TP}$ were significantly higher than those with a $6 \mathrm{TP}$ score of $1-3$

\section{Discussion}

In the rehabilitation of masticatory and swallowing functions, to improve physical function, the recovery of sensory function is also desirable because of the importance of sensory feedback for motor control and learning [20-25]. With the aim of evaluating the relationships between tongue sensory and motor dysfunction and the goal of developing a rehabilitation method that targets recovery of the oral sensory function, we previously investigated and reported the ability of young adults and the elderly to recognize the size and shape of items by using the tongue [17]. The method in which 20 different shapes of test pieces are used is effective for detailed analysis of stereognostic ability of the tongue, but completing the test was difficult for the elderly. Therefore, for use in further research, such as assessment and treatment of a larger number of elderly and patients, a simpler method of evaluation is needed. Therefore, we examined whether or not the difference in oral stereognostic ability among young adults and the elderly who receive care or not could be detected by an assessment using only 6 test pieces, which were selected from the 20 test pieces.

The rationale for selection of the 6 test pieces was as follows: In a previous report [17], the test pieces were categorized into the following 6 groups: polygonal (No. 1 to 4), triangular (No. 5 to 7), star-shaped (No. 8 to 10), circular-shaped (No. 11, 12), convex-shaped with no corners and convex ends (No. 13 to 16), and concave-shaped pieces with no corners and a concave middle (No. 17 to 20). In that study, we also discussed in detail what kind of sensory information is needed to perform stereognosis, which can be decreased in the elderly. The results suggested that sensory information characteristic of the shapes in a specific group, the contact area between the tongue and test piece, the shape of the test objects with angles, and asymmetrical shape are clues for recognition of shapes. There have been several reports on clues for recognition of shapes [16,24,26,27]. Although the shapes and numbers of test pieces used in those reports differed from those in our research, similar clues for identification of shapes were suggested. Thus, we selected 6 test pieces on the basis of abilities to detect differences in simple but different shapes, similar but different-sized shapes, and similar shapes but with or without angles.

In addition to our study, there have been some reports that oral stereognostic ability of young adults is quite high $[11,12,25]$. For the 198 young adults in this study, the mean number of correct responses in the 20TP test was $16.6 \pm 2.2$. In the $6 \mathrm{TP}$ test, almost all of the subjects could identify all test pieces perfectly and only 10 subjects could not. Misidentification between No. 1 and 15 was the most frequent mistake; the total number of subjects who misidentified No. 1 as No. 15 was 4 and the total number of subjects who misidentified No. 15 as No. 1 was also 4. It was considered that they could not recognize whether or not the test piece had angles because the contact area between the tongue and the test piece was small. There were subjects who could not recognize simple forms like No. $3(n=3)$ and No. $13(n=1)$. They seemed to have trouble as a result of the large contact area. Our study and others have also reported that oral stereognostic ability decreases with age [2631]. The ratio of elderly who do not need care and could identify all test pieces perfectly was significantly lower than that of young adults. Most cases of misidentification were the same as seen in the young adults: misidentification between No. 1 and 15. The total numbers of elderly subjects who misidentified No. 1 as No. 15 and misidentified No. 15 as No. 1 were 20 and 5, respectively. This misidentification was remarkably increased compared to the group of young adults. The second most common misidentification was that of No. 13 as No. $3(n=8)$. About 70 percent $(n=22)$ of 34 subjects who misidentified test pieces in the 6TP test made these mistakes described above. They seemed unable to determine whether or not there were angles in simple shapes. Most subjects who misidentified more than 2 pieces made mistakes in identification of other shapes in addition to the 2 misidentifications described above. It has been reported that many elderly receiving care have masticatory or swallowing dysfunction [32]. Even in our subjects receiving care without eating or swallowing dysfunction, although the sample size was small compared to the other 2 groups, the ratio of subjects who could identify all test pieces was much lower than that in the elderly who did not need care. Most subjects misidentified more than one piece. The variety in the misidentification also increased, and in most misidentifications, inferring the cause of these is difficult.

In 35 elderly who were examined in both the 6TP and 20TP tests, the number of correct responses in the $6 \mathrm{PT}$ test had a significant positive correlation to those in the $20 \mathrm{PT}$ test $(\mathrm{p}<0.001$ by one-way ANOVA). This result indicated that the decrease in stereognostic ability of the tongue could be detected by the 6TP test. The reasons why the test was conducted only once were to exclude any learning effect with the small number of test pieces and also in consideration of the elderly subjects' attitude, which was not refusal but nor was it positive toward the test. Even in the one-time test, we detected a large difference in the ratio of subjects who could identify all 6 shapes: about $95 \%$ of young adults and about $44 \%$ of healthy elderly. Moreover, there was a high correlation between the number of correct responses in the $6 \mathrm{PT}$ and 20PT tests among the elderly subjects.

Oral functions in patients with stroke and Parkinson's disease have been studied [33-35]. Reduced oral sensations or cognitive function in patients with stroke, dementia, and neurodegenerative diseases might cause inability to feel or localize the food in the mouth and may further cause prolongation of the oral transit time or result in residual food in the oral cavity [36,37]. However, the relationship between oral stereognostic ability and oropharyngeal functions, including oral preparatory phase, oral transport phase or pharyngeal phase, has not been investigated in these patients. Similar to the elderly subjects, tolerating complicated assessment is generally difficult for these patients due to their difficulties in cognitive function or endurance. In the future, this oral stereognostic ability test using 6 test pieces may have potential for clinical applications due to its convenience. In addition, reduction of test pieces may assist in recent oral rehabilitation approaches for improvement of oral stereognostic ability in elderly patients. Previously, for elderly in their 80 's, we suggested the possibility of improving their stereognostic ability through training using 20 test pieces, and a significant difference was observed regarding the number of correct responses between before and after the training [17]. It may be beneficial for elderly or patients with difficulties in oral stereognostic ability or oropharyngeal dysphagia to use these 6 test pieces as training after adjustments in number, i.e. gradual increases from easier to more difficult according to individual oral stereognostic ability score. 
Citation: Kawagishi S, Tanaka T, Shimodozono M, Yoshino K (2013) Simplifying the Assessment of Stereognostic Ability of the Tongue in Elderly Subjects Using Six Selected Test Pieces. Aging Sci 1: 111. doi: 10.4172/2329-8847.1000111

\section{Acknowledgement}

This research was partially supported by a Grant-in-Aid for Scientific Research (No. 21500487) from the Japan Society for the Promotion of Science.

\section{References}

1. Gordon C, Hewer RL, Wade DT (1987) Dysphagia in acute stroke. Br Med J (Clin Res Ed) 295: 411-414

2. Foley NC, Martin RE, Salter KL, Teasell RW (2009) A review of the relationship between dysphagia and malnutrition following stroke. J Rehabil Med 41: 707713

3. Kim JS, Choi-Kwon S, Kwon SU, Lee HJ, Park KA, et al. (2005) Factors affecting the quality of life after ischemic stroke: young versus old patients. J Clin Neurol 1: 59-68.

4. Miura H, Yamasaki K, Morizaki N, Moriya S, Sumi Y (2010) Factors influencing oral health-related quality of life (OHRQoL) among the frail elderly residing in the community with their family. Arch Gerontol Geriatr 51: e62-65.

5. Campbell-Taylor I (2008) Oropharyngeal dysphagia in long-term care: misperceptions of treatment efficacy. J Am Med Dir Assoc 9: 523-531.

6. Logemann JA (1998) Evaluation and treatment of swallowing disorders.

7. Han TR, Paik NJ, Park JW (2001) Quantifying swallowing function after stroke: A functional dysphagia scale based on videofluoroscopic studies. Arch Phys Med Rehabil 82: 677-682.

8. Han TR, Paik NJ, Park JW, Kwon BS (2008) The prediction of persistent dysphagia beyond six months after stroke. Dysphagia 23: 59-64.

9. Nishiwaki K, Tsuji T, Liu M, Hase K, Tanaka N, et al. (2005) Identification of a simple screening tool for dysphagia in patients with stroke using factor analysis of multiple dysphagia variables. J Rehabil Med 37: 247-251.

10. Schmidt RA (2008) Wrisberg CA. Motor Learning and Performance. (4th edn). Human Kinetics Publishers, Champaign, IL.

11. Jacobs R, Bou Serhal C, van Steenberghe D (1998) Oral stereognosis: a review of the literature. Clin Oral Investig 2: 3-10.

12. Grasso JE, Catalanatto FA (1979) The effects of age and full palatal coverage on oral stereognostic ability. J Prosthet Dent 41: 215-219.

13. Berry DC, Mahood M (1966) Oral stereognosis and oral ability in relation to prosthetic treatment. Br Dent J 120: 179-185.

14. Jacobs R, Bou Serhal C, van Steenberghe D (1997) The stereognostic ability of natural dentitions versus implant-supported fixed prostheses or overdentures. Clin Oral Investig 1: 89-94.

15. Müller F, Link I, Fuhr K, Utz KH (1995) Studies on adaptation to complete dentures. Part II: Oral stereognosis and tactile sensibility. J Oral Rehabil 22: 759-767.

16. Boliek CA, Rieger JM, Li SY, Mohamed Z, Kickham J, et al. (2007) Establishing a reliable protocol to measure tongue sensation. J Oral Rehabil 34: 433-441.

17. Kawagishi S, Kou F, Yoshino K, Tanaka T, Masumi S (2009) Decrease in stereognostic ability of the tongue with age. J Oral Rehabil 36: 872-879.

18. Mason EJ (1967) Studies on oral perception in involving subjects with alternations in anatomy and physiology. In: Second symposium on oral sensation and perception (ed. by Bosma, J.F). Charles C. Thomas, Springfield, Illinois, 295-301.

19. Kawagishi S, Kou F, Yoshino K, Amano N (2007) The stereognostic ability of the tongue in young adults (In Japanese, English abstract). J Kyushu Den Soc 61: $16-22$

20. Engelen L, van der Bilt A, Bosman F (2004) Relationship between ora sensitivity and masticatory performance. J Dent Res 83: 388-392.

21. Kaiba Y, Hirano S, Hayakawa I (2006) Palatal coverage disturbance in masticatory function. J Med Dent Sci 53: 1-6.
22. Müller F, Hasse-Sander I, Hupfauf L (1995) Studies on adaptation to complete dentures. Part I: Oral and manual motor ability. J Oral Rehabil 22: 501-507.

23. Yan C, Ye L, Zhen J, Ke L, Gang L (2008) Neuroplasticity of edentulous patients with implant-supported full dentures. Eur J Oral Sci 116: 387-393.

24. Hirano K, Hirano S, Hayakawa I (2004) The role of oral sensorimotor function in masticatory ability. J Oral Rehabil 31: 199-205.

25. Landt H, Fransson B (1975) Oral ability to recognize forms and oral muscula coordination ability in dentulous young and elderly adults. J Oral Rehabil 2 : 125-138.

26. Mantecchini G, Bassi F, Pera P, Preti G (1998) Oral stereognosis in edentulous subjects rehabilitated with complete removable dentures. J Oral Rehabil 25 185-189.

27. Ikebe K, Amemiya M, Morii K, Matsuda K, Furuya-Yoshinaka M, et al. (2007) Comparison of oral stereognosis in relation to age and the use of complete dentures. J Oral Rehabil 34: 345-350.

28. Litvak H, Silverman SI, Garfinkel L (1971) Oral stereognosis in dentulous and edentulous subjects. J Prosthet Dent 25: 139-151.

29. Smith PW, McCord JF (2002) Oral stereognostic ability in edentulous and dentate individuals. Eur J Prosthodont Restor Dent 10: 53-56.

30. Calhoun KH, Gibson B, Hartley L, Minton J, Hokanson JA (1992) Age-related changes in oral sensation. Laryngoscope 102: 109-116.

31. Ikebe K, Amemiya M, Morii K, Matsuda K, Furuya-Yoshinaka M, et al. (2007) Association between oral stereognostic ability and masticatory performance in aged complete denture wearers. Int J Prosthodont 20: 245-250.

32. Yamasaki M (2009) Prevalence of aspiration pneumonia. General rehabilitation 7:105-109.

33. Pow EH, Leung KC, McMillan AS, Wong MC, Li LS, et al. (2001) Ora stereognosis in stroke and Parkinson's disease: a comparison of partially dentate and edentulous individuals. Clin Oral Investig 5: 112-117.

34. Leung KC, Pow EH, McMillan AS, Wong MC, Li LS, et al. (2002) Oral perception and oral motor ability in edentulous patients with stroke and Parkinson's disease. J Oral Rehabil 29: 497-503.

35. Bakke M, Larsen SL, Lautrup C, Karlsborg M (2011) Orofacial function and ora health in patients with Parkinson's disease. Eur J Oral Sci 119: 27-32.

36. André JM, Beis JM, Morin N, Paysant J (2000) Buccal hemineglect. Arch Neurol 57: 1734-1741.

37. Teismann IK, Steinstraeter O, Stoeckigt K, Suntrup S, Wollbrink A, et al. (2007) Functional oropharyngeal sensory disruption interferes with the cortical control of swallowing. BMC Neurosci 8: 62. 